\title{
Utilidad de la reacción de polimerasa en cadena convencional para la detección de Mycoplasma hominis, Ureaplasma spp. y Trichomonas vaginalis en muestras genitales de mujeres en consulta ambulatoria
}

\author{
Gonzalo Alarcón, Gabriela Barraza, Andrea Vera, Aniela Wozniak y Patricia García
}

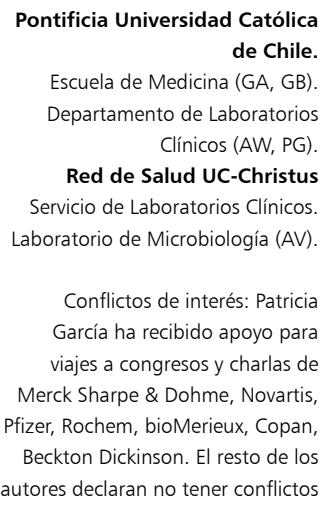

Pontificia Universidad Católica de Chile.

Escuela de Medicina (GA, GB)

Departamento de Laboratorios Clínicos (AW, PG)

Red de Salud UC-Christus Servicio de Laboratorios Clínicos. Laboratorio de Microbiología (AV).

Conflictos de interés: Patricia

García ha recibido apoyo para viajes a congresos y charlas de Merck Sharpe \& Dohme, Novartis, Pfizer, Rochem, bioMerieux, Copan, Beckton Dickinson. El resto de los autores declaran no tener conflictos

de interés.

Fuentes de financiamiento:

Fondo SENTRY Laboratorio de Microbiología, Departamento de Laboratorios Clínicos, Red de Salud UC-Christus

Recibido: 7 de mayo de 2015 Aceptado: 6 de octubre de 2015

Correspondencia a: Patricia García Cañete pgarcia@med.puc.c

\section{Introducción}

Usefulness of conventional polymerase chain reaction for the detection of Mycoplasma hominis, Ureaplasma spp. and Trichomonas vaginalis in female outpatient's genital samples

Introduction: Trichomonas vaginalis, Mycoplasma hominis and Ureaplasma spp. are microorganisms responsible for genitourinary and pregnancy pathologies. Nucleic acid amplification methods have shown several advantages, but have not been widely studied for the detection of these microorganisms. Aim: To implement a conventional polymerase chain reaction (PCR) for the detection of the microorganisms and to compare its results versus the methods currently used at our laboratory. Material and Methods: 91 available samples were processed by PCR, culture (M. hominis y Ureaplasma spp.) and wet mount (T. vaginalis). Results were compared and statistically analyzed by kappa agreement test. Results: 85,80 and 87 samples resulted in agreement for the detection of $M$. hominis, Ureaplasma spp. y T. vaginalis, respectively. For M. hominis and Ureaplasma spp., agreement was substantial, whereas for T. vaginalis it was moderate, however, for the latter, PCR detected more cases than wet mount. Conclusion: We recommend the implementation of PCR for detection of $T$. vaginalis whereas culture kit is still a useful method for the other microorganisms.

Key words: Mycoplasma hominis, Ureaplasma spp., Trichomonas vaginalis, polymerase chain reaction, DNA, culture.

Palabras clave: Mycoplasma hominis, Ureaplasma spp., Trichomonas vaginalis, reacción de polimerasa en cadena, ADN, cultivo.
$T$ richomonas vaginalis, Mycoplasma hominis, Ureaplasma parvum y Ureaplasma urealyticum son microorganismos causantes de infecciones de transmisión sexual (ITS), patologías genito-urinarias y del embarazo, en mujeres ${ }^{1,2}$.

Trichomonas vaginalis es un protozoo flagelado cuyo único hospedero conocido es el ser humano, ocupando el tracto genito-urinario como nicho ${ }^{3}$. Corresponde a una de las causas más frecuentes de ITS en el mundo y su presentación más habitual es la vaginitis, que puede variar desde una infección asintomática a un proceso inflamatorio pelviano grave $e^{1,3}$. Además, se ha asociado a pérdida de la fertilidad, patologías del embarazo y aumento del riesgo de infección por $\mathrm{VIH}^{4}$.

Mycoplasma hominis, U. parvum y U. urealyticum son bacterias pertenecientes a la familia Mycoplasmataceae, filogenéticamente derivadas de bacterias grampositivas, que característicamente carecen de pared celular ${ }^{5,6}$.
Estos microorganismos se han asociado principalmente a patologías del embarazo, incluyendo corio-amnionitis, rotura prematura de membranas y abortos espontáneos, entre otras ${ }^{7}$. Por su parte, $M$. hominis se ha relacionado con vaginosis bacteriana y proceso inflamatorio pélvico, mientras que Ureaplasma spp. se ha descrito como causante de uretritis en mujeres ${ }^{5,13}$.

Ureaplasma parvum y $U$. urealyticum son dos especies de importancia humana que en un comienzo eran consideradas como biovares de la especie $U$. urealyticum $^{5,8}$. El biovar 1 fue separado constituyendo la especie $U$. parvum, mientras que el biovar 2 , antes denominado T960, mantuvo la nominación $U$. urealyticum. Ambas especies son indistinguibles fenotípicamente, pudiendo ser diferenciadas mediante reacción de polimerasa en cadena (RPC) para el segmento $16 \mathrm{~S}$ de ARN ribosomal ${ }^{8}$.

Por otra parte, se ha demostrado asociación entre T. vaginalis y $M$. hominis, reportándose hasta $71 \%$ de co-infección. Se propone como explicación una relación 
simbiótica entre ambos microorganismos, dada la capacidad de $M$. hominis de infectar a T. vaginalis $^{9,13}$.

Actualmente, la Sociedad Americana de Enfermedades Infecciosas (IDSA) reconoce como métodos de detección para $T$. vaginalis la microscopia al fresco, el test rápido de antígeno, hibridación de ADN, cultivo y test APTIMA de amplificación de ácidos nucleicos (Gen-Probe ${ }^{\circledR}$, Inc., San Diego, CA $)^{10}$. Entre ellos, el cultivo se considera el patrón de oro, siendo su sensibilidad de $85-95 \%$ y su especificidad de $95-100 \%$. En el Laboratorio de Microbiología de la Pontificia Universidad Católica de Chile, el método utilizado es la microscopia al fresco, para el que se reporta una sensibilidad de 50-60\% y una especificidad de $90 \%$ en poblaciones de alta prevalencia y con personal especializado en microscopia ${ }^{3,4}$.

En el caso de $M$. hominis y el género Ureaplasma, los métodos posibles de detección descritos son el cultivo anaerobio o aerobio y kits comerciales de cultivo ${ }^{10,13}$. En nuestro laboratorio, se utiliza el kit comercial de cultivo Mycofast ${ }^{\circledR}$ EvolutioN (Ref. 00060, EliTechGroupSolutions ${ }^{\circledR}$, Puteaux, Francia), cuyo fabricante declara una sensibilidad de 98,2\% y una especificidad de $100 \%$ para muestras uretrales y vaginales ${ }^{11}$. Si bien IDSA no menciona como método de detección las pruebas de amplificación de ácidos nucleicos, Petrikkos y cols., proponen el uso de la RPC convencional con una sensibilidad aproximada para $M$. hominis y U. urealyticum de 96 y $94 \%$ y una especificidad de 87 y $92 \%$, respectivamente $^{12}$. Debe considerarse para la evidencia antes expuesta que en el período de publicación del artículo, $U$. urealyticum incluía sin distinción los dos biovares que ahora constituyen especies.

Dado que en la actualidad los métodos de amplificación de ácidos nucleicos presentan notables ventajas por sobre los métodos de cultivo, los objetivos de este trabajo fueron implementar la RPC para la detección de T. vaginalis, $M$. hominis y Ureaplasma spp. y evaluar la concordancia entre esta metodología y las pruebas disponibles en nuestro laboratorio.

\section{Material y Métodos}

Se procesaron 91 muestras consecutivas de mujeres para quienes se había solicitado el estudio de T. vaginalis, M. hominis y Ureaplasma spp. en secreción vaginal o cervical. Las muestras fueron recibidas y procesadas en el Laboratorio de Microbiología de la Red de Salud UC-CHRISTUS en los períodos febrero-marzo y juniooctubre del año 2013.

\section{Métodos microbiológicos convencionales}

Consistieron en cultivo aeróbico para M. hominis y Ureaplasma spp. mediante el kit Mycofast ${ }^{\circledR}$ EvolutioN
(EliTechGroupSolutions ${ }^{\circledR}$, Puteaux, Francia) y por microscopia de la muestra al fresco para $T$. vaginalis.

\section{Reacción de polimerasa en cadena}

La extracción de ADN de las muestras se realizó mediante el kit QIAmp ${ }^{\circledR}$ DNA Mini (Qiagen ${ }^{\circledR}$, Limburgo, Holanda) y se almacenaron, hasta su procesamiento, a $-20^{\circ} \mathrm{C}$.

Se implementó una RPC convencional para la detección de T. vaginalis, M. hominis y Ureaplasma spp., utilizando los partidores propuestos por Díaz y cols. ${ }^{13}$ Para T. vaginalis se utilizó el par TvA5/TvA6 (amplifica un segmento de $102 \mathrm{pb}$ que codifica para la proteína $\mathrm{G} 3$ ), con temperatura de annealing (Ta) de $48^{\circ} \mathrm{C}$. Para M. hominis se utilizó el par RNAH1/RNAH2 (amplifica un segmento de $334 \mathrm{pb}$ del $16 \mathrm{~S}$ ARNr) con Ta de $50^{\circ} \mathrm{C}$. Y para Ureaplasma spp. se utilizó el par Uu1/Uu2 (amplifica un segmento de $541 \mathrm{pb}$ del gen de la ureasa) con Ta de $50^{\circ} \mathrm{C}$.

La RPC fue realizada usando una mezcla de reacción constituida por $1 \mathrm{x}$ de tampón Green GoTaqFlexi (Promega ${ }^{\circledR}$, Madison, WI), 1 mMde $\mathrm{MgCl}_{2}$ (Promega ${ }^{\circledR}$, Madison, WI), 0,13 mM de cada deoxi-nucleótido (ThermoScientific ${ }^{\circledR}$, Waltham, MA), 0,6 $\mu \mathrm{M}$ de cada partidor correspondiente, $0,04 \mathrm{U} / \mu \mathrm{l}$ de Taq polimerasa (Promega ${ }^{\circledR}$, Madison, WI) y $5 \mu \mathrm{l}$ de ADN extraído de cada muestra. El volumen final de la reacción fue de $25 \mu$ por cada alícuota. El programa utilizado en el termociclador Veriti 96 Well Thermal Cycler (Applied Biosystems ${ }^{\circledR}$, Foster City, CA) fue de $94^{\circ} \mathrm{C}$ por $5 \mathrm{~min}$, seguido de 35 ciclos $\left(94^{\circ} \mathrm{C}\right.$ por $40 \mathrm{~s}$, Ta por $40 \mathrm{~s} \mathrm{y} 72^{\circ} \mathrm{C}$ por $\left.1 \mathrm{~min}\right)$, seguidos por una extensión final de 8 min a $72^{\circ} \mathrm{C}$. La detección de los productos de RPC se realizó en geles de agarosa al 1,5\% utilizando un estándar de ADN (100 pb, Invitrogen ${ }^{\circledR}$, Waltham, MA).

Como control positivo se utilizó el ADN cuantificado del patógeno correspondiente (M. hominis y T. vaginalis: Vircell Microbiologists ${ }^{\circledR}$, Granada, España y U. urealyticum: Minerva Biolabs ${ }^{\circledR}$, Berlín, Alemania) y como control negativo se utilizó tampón de elución (AE) del kit de extracción QIAmpDNA Mini. Para controlar la eficiencia de la extracción, así como la ausencia de inhibidores en la reacción, se utilizó como control interno el gen de la $\beta$-globina humana (IDT ${ }^{\circledR}$, Coralville, IA).

Se realizaron los estudios de sensibilidad analítica para cada RPC y el estudio de concordancia mediante el test kappa de Cohen ( $\kappa)$, con un intervalo de confianza (IC) al $95 \%{ }^{14}$. Los resultados del análisis fueron evaluados según el criterio expuesto en Landis y cols., considerando un valor de $\kappa$ entre 0 y 1 , donde 1 indica máxima concordancia ${ }^{14}$. La escala cualitativa es la siguiente: pobre $(<0,00)$, leve $(>0,00-0,20)$, aceptable $(0,21-0,40)$, moderada $(0,41-0,60)$, considerable $(0,61$ $0,80)$ y casi perfecta $(0,81-1,00)$. 
Tabla 1. Comparación entre métodos para la detección de Mycoplasma hominis, Ureaplasma spp. y Trichomonas vaginalis

\begin{tabular}{|c|c|c|c|c|}
\hline \multirow[t]{2}{*}{ Mycoplasma hominis } & & \multicolumn{3}{|c|}{ Cultivo Mycofast ${ }^{\circledR}$ Evolution } \\
\hline & & Positivo & Negativo & Total \\
\hline \multirow{3}{*}{$\begin{array}{l}\text { Reacción de polimerasa } \\
\text { en cadena }\end{array}$} & Positivo & 6 & 2 & 8 \\
\hline & Negativo & 4 & 79 & 83 \\
\hline & Total & 10 & 81 & 91 \\
\hline Ureaplasma spp & & \multicolumn{3}{|c|}{ Cultivo Mycofast ${ }^{\circledR}$ Evolution } \\
\hline \multirow{4}{*}{$\begin{array}{l}\text { Reacción de polimerasa } \\
\text { en cadena }\end{array}$} & & Positivo & Negativo & Total \\
\hline & Positivo & 29 & 7 & 36 \\
\hline & Negativo & 4 & 51 & 55 \\
\hline & Total & 33 & 58 & 91 \\
\hline Trichomonas vaginalis & & \multicolumn{3}{|c|}{ Microscopia al fresco } \\
\hline \multirow{4}{*}{$\begin{array}{l}\text { Reacción de polimerasa } \\
\text { en cadena }\end{array}$} & & Positivo & Negativo & Total \\
\hline & Positivo & 2 & 4 & 6 \\
\hline & Negativo & 0 & 85 & 85 \\
\hline & Total & 2 & 89 & 91 \\
\hline
\end{tabular}

\section{Resultados}

Las concentraciones mínimas detectadas (sensibilidad analítica) fueron $1,19 \times 10^{-5} \mathrm{ng} / \mu \mathrm{L}$ para $M$. hominis, $1 \times$ $10^{-4} \mathrm{ng} / \mu \mathrm{L}$ para Ureaplasma spp. y $0,488 \mathrm{ng} / \mu \mathrm{L}$ para $T$. vaginalis.

Para la detección de $M$. hominis, se encontró que en 85 de las 91 muestras procesadas se obtuvieron resultados concordantes entre los métodos (Tabla 1), siendo dos muestras positivas para cultivo, negativas por RPC. Se calculó un valor kappa de 0,631 (IC: 0,359-0,902), correspondiente a concordancia considerable.

Para Ureaplasma spp., en 80 de las 91 muestras se encontraron resultados concordantes (Tabla 1), resultando tres muestras positivas por cultivo, negativas por RPC, con un valor kappa calculado de 0,744 (IC: 0,602-0,885), que corresponde a un acuerdo considerable.

En el caso de T. vaginalis, la RPC determinó seis muestras como positivas versus sólo dos por la microscopía al fresco (Tabla 1), existiendo concordancia en estas últimas. En su totalidad, 87 de las 91 muestras mostraron resultados concordantes. Todas las discordancias correspondieron a muestras categorizadas como negativas por microscopía al fresco con resultado positivo mediante RPC. El valor kappa calculado fue 0,483 (IC: 0,059$0,907)$, que corresponde a concordancia moderada.

\section{Discusión}

Considerando las ventajas que han mostrado los métodos de amplificación de ácidos nucleicos, el presente estudio busca determinar la utilidad de la RPC como método de detección de microorganismos causantes de patología genito-urinaria femenina: M. hominis, $U$. parvum, U. urealyticum y $T$. vaginalis.

Para la detección de $M$. hominis y Ureaplasma spp., si bien se encontró que la concordancia entre las técnicas era considerable, destaca que el kit de cultivo detectó más casos que la RPC. Esto indicaría que si, efectivamente, los resultados positivos en el kit de cultivo definieran la infección, los resultados negativos de la RPC constituirían falsos negativos. Lo anterior no se puede asegurar, ya que el kit de cultivo no ha sido reconocido como patrón de oro. Por otro lado, la existencia de variables no investigadas, como degradación de ADN, pueden haber afectado los resultados de la RPC. Considerando además el menor costo y la experiencia del personal del laboratorio, el kit de cultivo continuaría siendo el método más recomendable para la detección de ambos microorganismos.

En cuanto a $T$. vaginalis, la concordancia entre la RPC y la microscopia al fresco fue moderada. En términos estadísticos, este nivel de concordancia podría ser explicado por la mayor cantidad de muestras negativas que son concordantes; sin embargo, cabe destacar que la RPC detectó una mayor cantidad de muestras positivas. Dada las dificultades técnicas de la microscopia al fresco, incluyendo la necesidad de un operador especializado y de un rápido e inmediato procesamiento para mantener viable al microorganismo y considerando la baja sensibilidad reportada en la literatura científica ${ }^{3}$, consideramos recomendable la implementación de RPC en la detección de $T$. vaginalis.

Es necesario continuar la evaluación considerando test de amplificación de ácidos nucleicos con metodologías de mayor sensibilidad analítica como RPC en tiempo real, entre otras.

Agradecimientos. Los autores agradecen a la alumna de Licenciatura en Biología, Srta. Camila Tiznado por su colaboración en la etapa inicial de la implementación de los métodos moleculares.

\section{Resumen}

Introducción: Trichomonas vaginalis, Mycoplasma hominis y Ureaplasma spp. son microorganismos causantes de patología genito-urinaria y durante el embarazo. Los métodos de amplificación de ácidos nucleicos han demostrado numerosas ventajas, pero no han sido ampliamente estudiados para la detección de estos microorganismos. Objetivo: Implementar una reacción de polimerasa en cadena convencional (RPC) para su detección y comparar sus resultados con los métodos actuales de nuestro laboratorio. Material y Métodos: Se procesaron 91 muestras 
mediante RPC, cultivo (M. hominis y Ureaplasma spp.) y observación microscópica al fresco (T. vaginalis). Los resultados fueron comparados y analizados estadísticamente mediante el test de concordancia kappa. Resultados: 85 , 80 y 87 muestras tuvieron resultados concordantes para la detección de M. hominis, Ureaplasma spp. y T. vaginalis, respectivamente. Para M. hominis y Ureaplasma spp. el nivel de concordancia fue considerable mientras que para $T$. vaginalis fue moderado; sin embargo, para esta última, la RPC detectó más casos que la microscopia al fresco. Conclusión: Se recomienda la implementación de la RPC para la detección de T. vaginalis. Para M. hominis y Ureaplasma spp. el kit de cultivo continúa siendo un buen método.

\section{Referencias bibliográficas}

1.- McCormack W M, Rein M F. Chapter 102: Urethritis. Mandell G L, Bennett J E, Dolin R, editors. Mandell, Douglas and Bennett's. Principles and Practice of Infectious Diseases, 6th ed. Philadelphia: Churchill Livingstone Elsevier; 2005, p. 1347-57.

2.- Kenny G E. Chapter 182: Genital Mycoplasmas: Mycoplasma genitalium, Mycoplasma hominis and Ureaplasma spp. Mandell G L, Bennett J E, Dolin R, editors. Mandell, Douglas and Bennett's. Principles and Practice of Infectious Diseases, 6th ed. Philadelphia: Churchill Livingstone Elsevier; 2005, p. 2280-1357.

3.- Harp D F, Chowdhury I. Trichomoniasis: evaluation to execution. Eur J Obstet Gynecol Reprod Biol 2011; 157: 3-9.

4.- $\quad$ Eckert L O. Acute vulvovaginitis. N Engl J Med 2006; 355: 1244-52.

5.- Taylor-Robinson D. Infections due to species of Mycoplasma and Ureaplasma: an update. Clin Infect Dis 1996; 23: 671-82.

6.- Ryan K J, Ray C G. Mycoplasma. Ryan KJ,
Ray CG. Sherris Medical Microbiology. $6^{\text {th }}$ Ed. New York: McGraw-Hill; 2014.

7.- Larsen B, Hwang J. Mycoplasma, Ureaplasma, and adverse pregancy outcomes: a fresh look. Infect Dis Obstet Gynecol 2010; 2010: pii521921

8.- Martínez M A, Ovalle A, Santa-Cruz A, Barrera B, Vidal R, Aguirre R. Occurrence and antimicrobial ausceptibility of Ureaplasma parvum (Ureaplasma urealyticum biovar 1) and Ureaplasma urealyticum (Ureaplasma urealyticum biovar 2) from patients with adverse pregnancy outcomes and normal pregnant women. Scand J Infect Dis 2001; 33: 604-10.

9.- Dessi D, Rapelli P, Díaz N, Cappuccinelli P, Fiori P L. Mycoplasma hominis and Trichomonas vaginalis: a unique case of symbiotic relationship between two obligate human parasites. Front Biosci 2006; 11: 202834.

10.- Baron E J, Miller J M, Weinstein M P, Richter S S, Gilligan P H, Thomson R B Jr, et al. A guide to utilization of the microbiology laboratory for diagnosis of infectious diseases:
2013 Recommendations by the Infectious Diseases Society of America (IDSA) and the American Society for Microbiology (ASM). Clin Infect Dis 2013; 57: e22-e121.

11.- Grattard F, Soleihac B, De Barbeyrac B, Bebear C, Seffert P, Pozzeto B. Epidemiologic and molecular investigations of genital mycoplasmas from women and neonates at delivery. Pediatr Infect Dis J 1995; 14: 853-8.

12.- Petrikkos G L, Hadjisoteriou M, Daikos G L. PCR versus culture in the detection of vaginal Ureaplasma urealyticum and Mycoplasma hominis. Int J Gynaecol Obstet 2007; 97 : 202-3.

13.- Díaz N, Dessi D, Dessole S. Fiori P, Rapelli P. Rapid detection of coinfections by Trichomonas vaginalis, Mycoplasma hominis and Ureaplasma urealyticum by a new multiplex polymerase chain reaction. Diagn Microbiol Infect Dis 2010; 67: 30-6.

14.- Landis J R, Koch G G. The measurement of observer agreement for categorical data. Biometrics 1977; 33: 159-74. 